\section{A microcomputer program to demonstrate simple operant reinforcement schedules}

\author{
JOHN WALKENBACH \\ University of Montana, Missoula, Montana 59812
}

Basic schedules of reinforcement are taught in nearly every introductory psychology course. Although reinforcement contingencies appear simple, they are sometimes hard for the students to grasp; a firm understanding of them is necessary, since they form the basis for more advanced procedures.

A typical teaching demonstration strategy involves training laboratory rats to press a lever for food reinforcers and observing the rate of this behavior as a function of the reinforcement contingency (e.g., fixed ratio, variable interval, etc.). Rate of responding is a standard data source and is best shown by a cumulative record of responses. While this type of demonstration has the potential to be very instructive, it also has a number of drawbacks: Specialized equipment and trained personnel are often needed, a colony of animals must be maintained, and the process of food deprivation, shaping, and waiting for the behavior to stabilize is often time-consuming and tedious.

This paper describes an alternative to the traditional animal demonstration. The computer program described here serves as an extremely flexible instructional aid for teaching the fundamentals of reinforcement schedules.

Description of the Program. The program runs on an Apple II microcomputer. When the program is executed, the user is presented a menu that lists seven options. Choices are made interactively, and any of the following schedules may be run: fixed ratio, variable ratio, fixed interval, and variable interval. The user is then asked for the ratio or interval value or the average value for the variable schedules.

The screen is cleared and a cumulative record is drawn using the Apple II high-resolution graphics capability (Figure 1). Responses are made by depressing the "space" bar on the keyboard. Receipt of a reinforcer is indicated using the standard format of a diagonal tick. An additional optional auditory signal may be specified to indicate reinforcement. Pressing the "escape" key at any time clears the screen and returns the user to the menu.

One of the program alternatives is to run a schedule that is unknown to the user. Choosing this option causes the computer to pick one of the four schedules and a value at random. This option is particularly useful in demonstrating the development of stable responding, and it can also be used to test the student's ability to identify a particular schedule. Before exiting from this option, the chosen schedule is revealed to the user.

The final option is to list a set of instructions on the screen. This simply provides a brief description of the
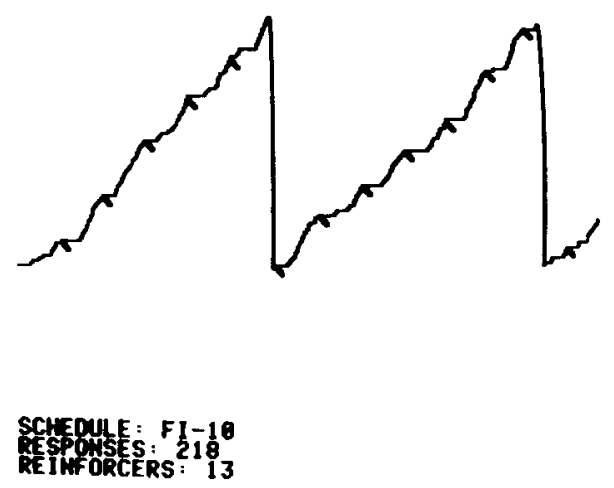

Figure 1. A sample of a cumulative record depicted using Apple II high-resoultion graphics.

schedules and instructs the user in how to run the program.

The program may be used in several ways: An instructor may simulate a rat's behavior under various schedules, or a student may be selected as a subject and instructed to maximize the reinforcers and minimize responding. This is most effective when the subject is not allowed to view the cumulative curve on the screen and reinforcement is signaled by the auditory cue. Using this blind procedure under fixed-interval schedules rapidly produces the characteristic postreinforcement pauses, which appear as scallops on the cumulative record.

Hardware Requirements. The program was written in APPLESOFT BASIC for the Apple II and uses less than $16 \mathrm{~K}$ bytes of random-access memory. No external clock is needed, since all timing is performed using calibrated loops. Therefore, the program will run on any minimally configured Apple computer with high-resolution graphics capability. Due to the dependence on many functions unique to APPLESOFT, conversion to other systems cannot be undertaken easily. One possible modification that can be performed relatively simply is to utilize the program in conjunction with external devices, such as levers and food dispensers. Thompson (1979) describes an inexpensive technique to allow such interfacing with the Apple II.

Availability. A listing of the program (approximately 170 lines) may be obtained at no cost by sending a large self-addressed stamped envelope to John Walkenbach, Montana University Affiliated Program, University of Montana, Missoula, Montana 59812.

\section{REFERENCE}

Thompson, G. C. Behavioral programming with the APPLE II microcomputer. Behavior Research Methods \& Instrumentation, 1979, 11, 585-588.

(Accepted for publication August 4, 1981.) 